\section{La Révolution française}

Cahiers de l'Institut d'histoire de la Révolution française

5 | 2013

Le républicanisme anglais dans la France des

Lumières et de la Révolution

\title{
La référence à l'Angleterre et au républicanisme anglais pendant le procès de Louis XVI
}

\section{Clizia Magoni}

\section{(2) OpenEdition}

Journals

Édition électronique

URL : http://journals.openedition.org//rf/962

DOI : $10.4000 /$ Irf.962

ISSN : 2105-2557

Éditeur

IHMC - Institut d'histoire moderne et contemporaine (UMR 8066)

Référence électronique

Clizia Magoni, « La référence à l'Angleterre et au républicanisme anglais pendant le procès de Louis XVI », La Révolution française [En ligne], 5 | 2013, mis en ligne le 31 décembre 2013, consulté le 19 avril 2019. URL : http://journals.openedition.org/Irf/962 ; DOI : 10.4000/Irf.962

Ce document a été généré automatiquement le 19 avril 2019

(c) La Révolution française 


\title{
La référence à l'Angleterre et au républicanisme anglais pendant le procès de Louis XVI
}

\author{
Clizia Magoni
}

1 Dès le début de l'instruction du procès de Louis XVI jusqu'à son exécution, la référence à l'histoire anglaise du XVII siècle, à la condamnation de Charles I ${ }^{\mathrm{er}}$, à la proclamation du Commonwealth et à l'ascension de Cromwell, représenta un passage attendu dans les discours des contemporains. C'était surtout la comparaison entre le procès de Charles Stuart de 1648-1649 et celui de Louis Capet qui revenait souvent dans les discours prononcés par les députés de la Convention nationale aussi bien que dans la presse ou dans les pamphlets publiés à cette époque. Cette référence au passé républicain anglais était nourrie de lectures des ouvrages historiques provenant des îles britanniques qui avaient été traduits en français dans la seconde moitié du XVIII siècle et au commencement de la Révolution. La pensée politique du républicanisme anglais était également récupérée à l'occasion du débat, Milton, défenseur de la cause républicaine, étant l'auteur de référence. Pourtant, cette manière de faire appel à l'exemple qui venait de l'histoire d'outre-Manche n'était pas univoque : le passé venait à l'appui pour plaider pour ou contre l'opportunité, la légitimité du jugement et ensuite l'exécution du ci-devant roi $^{1}$.

2 A la fin du mois de septembre 1792, quelques jours après l'installation de la Convention dans la Salle du Manège des Tuileries, le journal hebdomadaire fondé par Cérutti La Feuille villageoise, s'interrogeait sur le sort du prisonnier du Temple. «Le projet de faire, dès-à présent, le procès à la famille royale, seroit un plan funeste » lisait-on dans le numéro du 27 septembre 1792. "C'est l'avis de tous les anglois qui ont embrassé notre cause. “Un roi chassé, disent-ils, n'a plus de partisans; un roi tué se fait plaindre, et cette compassion donne des défenseurs à sa famille. Tarquin n'eut point de successeurs; Charles I ${ }^{\text {er }}$ en a encore ${ }^{2}$.» A l'opposé, un autre journal hebdomadaire, les Révolutions de Paris, au même moment, proposait à ses lecteurs une opinion différente vis-à-vis de l'opportunité de juger le roi détrôné. Dans un article paru en ouverture du numéro du 29 septembre, le 
rédacteur soutenait la nécessité du procès et de l'exécution de Louis XVI, « aussi-tôt après son jugement ", pour affermir dans toutes les âmes les principes de l'égalité. Les forfaits de Louis XVI étaient énumérés et comparés avec ceux commis par Charles $\mathrm{I}^{\mathrm{er}}$ afin de montrer que les mêmes causes engendraient les mêmes conséquences, à savoir la punition sur l'échafaud.

Un adage raisonnable est reçu en Angleterre: Rex major singulis; sed minor universis ${ }^{3}$. Ce principe établi, la personne d'un roi demeure sacrée pour quelques individus; mais elle cesse de l'être aux yeux de la généralité des citoyens. Ainsi Charles premier avoit blessé les intérêts de tous; il périt sur un échafaud pour avoir fait assassiner, par les mains de ses partisans, dans seize grandes batailles, 450000 citoyens anglais. De pareils meurtres méritoient bien le sort qu'il éprouva. Aussi les sans-culottes de Londres demandèrent-ils la mort du tyran, chacun d'eux ayant perdu, dans les sept ans de guerre civile, un père, un fils, un frère, ou un ami. Louis a forfait à l'intérêt général comme Charles premier. L'un a tenu la conduite d'un traître, et l'autre fit ouvertement la guerre au peuple, de qui il tenoit sa couronne. Le premier a déployé, à côté de ses crimes, une hypocrisie méprisable, une lâcheté insigne, le second fut un ambitieux scélérat et fanatique. Louis existe dans un siècle de raison et de Lumières. Charles exista dans un temps où la mode de disputer sur des sophismes théologiques échauffoit encore follement et divisoit les esprits, et où la différence des cultes, autorisant le nom injurieux d'hérétique, pouvoit irriter les sectes et leur mettre les armes à la main. Le ci-devant roi des Français avoit avoué que dans le peuple réside le droit du souverain. Charles Stuart prétendoit le contraire, sans avoir prêté aucun serment, puisqu'en 1649 la constitution britannique ne gouvernoit point encore l'Angleterre, alors uniquement pourvue de la grande charte de Jean-Sans-Terre ${ }^{4}$.

En avance sur le débat de la Convention, ces journaux, tout en abordant la question de l'opportunité de juger Louis XVI, présentaient des opinions opposées par le biais de l'exemple du régicide anglais. Le passé suggérait des enseignements différents selon le point de vue adopté pour l'observer: si l'on se fixait sur les conséquences du procès de Charles Stuart, la mort du roi d'Angleterre n'avait pas empêché le retour de la monarchie ; au contraire, si l'on observait les conditions préliminaires qui avaient amené au jugement et l'exécution du roi criminel, on pouvait constater les mêmes forfaits et donc on pouvait déjà envisager le même châtiment.

Dans une situation encore toute à définir de la part de la Convention nationale, cette approche visant à comparer l'histoire de France à celle d'Angleterre, s'exprima également par la récupération ad hoc de textes originaux de la moitié du XVII ${ }^{e}$ siècle. Au cours du mois d'octobre 1792, parut une réédition de l'Histoire entière et véritable du procès de Charles Stuard ${ }^{5}$, éditée pour la première fois à Londres en $1650^{6}$. Le texte, dans l'édition moderne, était présenté aux lecteurs, précédé par une Préface de l'éditeur qui indiquait le sens de la correcte réception de l'ouvrage: «La nouvelle édition des détails du Procès de Charles premier », lisait-on, "n'a point l'objet d'appeler les vengeances de la loi sur la tête du criminel Louis XVI ; mais cette pièce devenue très-rare se trouve aujourd'hui d'un extrême intérêt, par les rapprochemens qu'elle fournit entre ces deux rois et entre les deux peuples. " Si le jugement ne devait pas avoir la signification d'une vengeance, néanmoins la comparaison entre les crimes de Charles $\mathrm{I}^{\mathrm{er}}$ et ceux de Louis XVI attestait sans aucun doute la culpabilité du roi français. [...] Pourtant, lisait-on à la fin de la préface, «L'ambition et les projets de Cromwel ont jétté de l'odieux sur le Procès de Charles premier aux yeux des nations et de la postérité. Nous n'avons pas encore de Cromwel; c'est à la Convention nationale à veiller crainte qu'il n'y en ait un à s'élever. 
L'abolition de la royauté, la déclaration de la France en république nous rassurent ${ }^{7}$. La réédition de cet ouvrage devait donc, servir d'instrument de réflexion sur le procès à instruire et la Convention nationale devait prendre garde, en jugeant Louis XVI, de ne pas permettre qu'un nouveau Cromwell surgisse des cendres de la royauté abattue.

6 A l'opposé, toujours au cours de l'automne 1792, un fervent défenseur de la cause du roi, fit rééditer un recueil qui réunissait quatre pièces publiées entre 1649 et 1650 , sous le titre de Relation véritable de la mort cruelle et barbare de Charles I I ${ }^{e r}$, précédé par un avis au lecteur authentique de l'une de ces pièces (Sommaire de ce qui s'est passé de plus mémorable en Angleterre, depuis l'année 1640 jusques au premier Janvier 1650) ${ }^{8}$. Cet ouvrage eut une certaine fortune éditoriale, attestée par trois éditions consécutives. La troisième sortit à Paris sans doute dans la seconde partie du 1793, puisque le livre se terminait par le portrait de Charlotte Corday, héroïne de la cause royaliste. L'anonyme promoteur de l'édition de ce recueil s'avisa, pourtant trop tard de l'ambiguïté du texte, qui fournissait des arguments soit pour soit contre le roi, et fit ressortir après l'exécution de Louis XVI, le même ouvrage précédé cette fois-ci d'un autre texte intitulé L'Angleterre instruisant la France, Tableau historique et politique du règne de Charles $I^{e r}$ et de Charles II ${ }^{9}$. Dès la première page, on y voyait une gravure qui représentait l'Angleterre, sous forme d'une femme sans couronne, montrant du doigt à la France encore couronnée, la phrase «Lisez et tremblez ", contenue dans un livre ouvert qu'elle tenait dans ses mains. Dans la légende de l'image on pouvait lire: «Le 8 février 1649 je commis un grand crime. Prenez bien garde de suivre mon exemple. Si du Dieu de bonté vous voulez implorer la clémence, Ouvrez les cachots, et brisez les fers de l'innocence. » Décidément le rédacteur n'avait pas les idées trop claires par rapport aux différents acteurs de l'histoire anglaise ; après avoir raconté les bienfaits des souverains Stuart, Charles I ${ }^{\mathrm{er}}$ et surtout de Charles II, aussi bien que les événements relatifs au Rye House Plot, il terminait son histoire en affirmant que "Charles ouvrit toutes les sources de la félicité publique, et vivifia le corps politique [...] protégea les sciences et les arts. A sa voix, la philosophie répandit ses lumières et ses bienfaits, et le génie enfanta des miracles : la physique eut Newton; la politique et la législation, Hobbes, Sydney et $\operatorname{Locke}^{10} \ldots$ », le même Sidney qui avait trouvé la mort sur l'échafaud pour sa participation au Rhy House Plot contre Charles II.

7 Entretemps, la Convention nationale avait chargé le Comité de Législation de rédiger un rapport afin de résoudre les questions juridiques posées par le jugement du roi. Dans la séance du 27 octobre 1792, le porte-parole du Comité, Jean-Baptiste Mailhe, informait la Convention de l'état d'avancement des travaux. Au cours de cette brève intervention, le député toulousain voulut mettre en garde l'assemblée sur les enjeux du procès à instruire contre le prisonnier du Temple: "Vous savez comment on cherchera à calomnier la nation française sur les mesures qu'elle va prendre relativement au ci-devant roi ; vous savez combien on a calomnié la nation anglaise sur le jugement qu'elle prononça contre Charles Stuart, non parce qu'il fut condamné à mort, mais parce que, dans ce grand procès, les formes prescrites par les lois n'avaient pas été remplies; et voilà pourquoi les historiens, mêmes les plus philosophes, ont calomnié le peuple anglais sur ce qu'ils appelaient, non la punition, mais l'assassinat de Charles Stuart ${ }^{11}$.» Toute de suite après la fin de ce discours, pourtant, le député Philippe Rülh prit la parole pour rectifier ce que Mailhe venait de dire : «Mailhe vous a dit, citoyens, que la justice que le peuple anglais a faite de l'infâme Charles Stuart n'a été justifiée par aucun historien; il se trompe : John Milton, auteur du Paradis perdu, a fait la justification du peuple anglais; et dans son livre vous trouverez de fortes raisons pour condamner Louis $\mathrm{XVI}^{12}$.» 
8 Mailhe reprit cette argumentation lors de la lecture devant la Convention du rapport du Comité de Législation concernant le jugement, le 7 novembre suivant. Le roi était justiciable, premier point établit par le Comité, et il l'était par la Convention nationale. La question suivante abordée par le rapport concernait quelles formes fallait-il suivre. L'argumentation de la réponse procédait par l'analyse de l'exemple anglais. Sans doute Charles avait mérité la mort, affirmait le porte-parole Jean-Baptiste Mailhe ; « mais son supplice ne pouvait pas être ordonné que par la nation ou par un tribunal choisi par elle ». De fait, selon le Comité de Législation, le Parlement anglais, à l'occasion du procès du roi d'Angleterre, ne représentait pas la nation dans la plénitude de sa souveraineté mais juste dans la limite de ses fonctions déterminées par la constitution. Par conséquent il ne pouvait pas juger le roi. C'est pour cela qu'il aurait dû « faire ce qu'a fait en France le corps législatif; il devait inviter la nation anglaise à former une convention. [...] Ce n'est donc pas la violation des formes prescrites en Angleterre pour les jugements criminels, mais c'est le défaut d'un pouvoir national, c'est le protectorat, qui ont jeté sur le procès de Charles Stuart cet odieux qu'on trouve retracé dans les écrits les plus philosophiques ${ }^{13}$ . » Par les limites et les erreurs de l'exemple de l'histoire d'Angleterre, donc, le Comité de Législation avait fondé la légitimité de la Convention à juger la cause de Louis XVI.

Cette attention raisonnée et précise pour l'histoire d'Angleterre de la période de la guerre civile et pour la pensée politique du républicanisme anglais, notamment de Milton, évoqué à l'appui de l'action, n'était pas pourtant occasionnée par le jugement de Louis XVI dont on débattait dans la Convention aussi bien que dans l'espace public. Comme Mailhe l'avait répété à plusieurs reprises, les historiens, "même les plus philosophes" avaient présenté sous un mauvais jour le procès et condamnation de Charles Stuart, le Commonwealth et l'avènement de Cromwell. Cette décennie au milieu du XVII ${ }^{e}$ siècle représentait, dans le cours de l'histoire anglaise, un moment négatif par rapport à un modèle politique exemplaire qui s'était affirmé à la suite de la Glorious Revolution. C'était l'interprétation de Montesquieu dans l'Esprit des lois, par ailleurs cité par Mailhe dans le rapport du Comité de Législation ${ }^{14}$, mais surtout le récit historique de David Hume, l'un de ces historiens-philosophes également évoqués, auteur d'une History of Great Britain under the House of Stuart, publiée entre 1754 et 1757 et traduite en français en 1760 sous le titre Histoire de la maison de Stuart sur le trône d'Angleterre ${ }^{15}$. L'ouvrage connut en France une grande fortune et faisait même partie des livres que Louis XVI gardait au Temple ${ }^{16}$. Jacques Necker le citait aussi dans ses Réflexions sur le procès de Louis XVI qu'il publia au mois de novembre afin de plaider l'innocence du roi : "Qu'on lise dans l'histoire de la Maison de Stuart » écrivait Necker, "rédigée par un écrivain philosophe, l'impression convulsive que fit sur tous les cœurs la dernière catastrophe de l'infortuné Charles I. Qu'on y arrête, si l'on peut, son attention, et que l'on se demande ensuite si, dans le rapport de nos sentimens, un Roi n'est qu'un homme ; s'il n'est qu'un homme, surtout lorsqu'il fut si long-tems environné de notre amour, lorsqu'il fut si longtems le signe de tous nos liens. Ah! Qu'on lise le plus affreux des récits, et qu'on essaye ensuite de considérer sans émotion les idées funestes auxquelles on voudroit accoutumer la Nation Françoise. Oui, qu'on le lise cet affreux récit, et qu'on ose ensuite confier aux passions exaltées du moment présent, le jugement d'un Prince réduit par la fortune à l'abandon le plus absolu ${ }^{17}$. » Si les français connaissaient les vicissitudes anglaises d'après l'Histoire de David Hume, souhaitait l'ancien ministre de Louis XVI, ils ne commettraient pas un geste auquel ne pourrait suivre que le regret et le repentir. 
10 La narration du procès de Charles $\mathrm{I}^{\mathrm{er}}$ et de l'avènement de Cromwell par Hume, avait véhiculé dans les lecteurs français une image négative du Commonwealth britannique. Pourtant, l'histoire d'Angleterre avait connu un nouveau récit plus républicain par rapport à ces deux interprétations très lues de l'histoire et du modèle monarchique anglais, grâce à l'ouvrage de Catherine Macaulay The History of England from the Accession of James I to that of Brunswick Line, publié en huit volume entre 1763 et 1771. Apprécié par Brissot qui en avait parlé dans des termes enthousiastes aussi dans son Discours sur la question de savoir si le Roi peut être jugé de juillet $1791^{18}$, l'ouvrage fut traduit en français par initiative de Mirabeau ${ }^{19}$, et publié en automne 1791 lorsqu'ils parurent les premiers deux tomes sous le titre de Histoire d'Angleterre depuis l'avènement de Jacques Ier jusqu'à la révolution ${ }^{20}$. Dans le Moniteur du 14 février 1792, on lisait à propos de l'ouvrage de Macaulay: "On reconnaît aujourd'hui même en Angleterre, que dans la partie de l'histoire britannique qui contient la querelle du peuple avec ses rois, et dans laquelle le peuple fut le plus fort, comme il l'est toujours quand il veut l'être, le célèbre Hume a été, pour ainsi dire, partial à force de partialité. C'est un reproche qu'on ne peut faire à madame Macaulay. Ardente amie de la liberté, elle a envisagé sous leur véritable point de vue les attentats des Stuart contre la constitution anglaise, la connivence de la chambre des pairs, et la fermeté des communes pendant cette époque orageuse, qui s'étend depuis l'avènement de Jacques $\mathrm{I}^{\text {er }}$ jusqu'à l'abdication de Jacques II, dans un espace de quatrevingt-quatre années ${ }^{21}$.»

11 D'ailleurs, l'importance de l'Histoire de Macaulay dans les “temps présents“ était soulignée et avertie par le même Mirabeau dont l'opinion était reportée dans l'avis préliminaire de l'éditeur en ouverture du premier tome: "Cette traduction n'est pas dans nos circonstances, un ouvrage ordinaire. Il existe tant de points de contact et de rapport entre ces événemens, ces personnages et nous, qu'en se bornant à les indiquer dans de simples notes, on se trouvera faire l'histoire des deux révolutions ${ }^{22}$.»

Or, Mirabeau joua un rôle très important dans la diffusion de la pensée du républicanisme classique au début de la Révolution. C'est le cas des deux pamphlets politiques de John Milton, l'Aeropagitica de 1644, publié en 1788 sous le titre Sur la liberté de la presse, imité de l'anglois de Milton, et la Defensio pro populo anglicano, publiée en 1651 en latin et ensuite traduite en anglais ${ }^{23}$. Cette Défense du peuple anglais avait été commissionnée à l'auteur du Paradis perdu par le Parlement, en réponse de la Defensio regia pro Carolo I de Claude Saumaise, quelque temps après l'exécution de Charles $\mathrm{I}^{\mathrm{er}}$. Par cet ouvrage, Milton en affirmant que les Anglais n'avaient pas fait périr un roi juste, pacifique, dévoué, mais un ennemi un destructeur de son pays ( a Destroyer of his country ${ }^{24} »$ ), entendait démontrer que tous "les rois était incontestablement soumis aux lois comme les autres hommes ", que toute nation avait la liberté d'ériger le type de gouvernement qu'elle voulait et de le changer, que le peuple avait le droit de destituer un tyran et de le soumettre aux lois des hommes.

13 La première édition de la Defensio fut traduite en français en 1789 par Jean-Baptiste Salaville à la demande de Mirabeau, sous le titre Théorie de la royauté, d'après la doctrine de Milton, précédée d'une préface sur Milton et ses ouvrages ; la même traduction connut deux éditions successives, en 1790, sous le titre de Doctrine de Milton sur la royauté, d'après l'ouvrage intitulé: Défense du peuple anglais et en 1791 comme Théorie de la royauté, d'après les principes de Milton, avec sa Défense du peuple, par Mirabeau, en 179125. Comme déjà dans le cas d'autres traductions, le texte avait été largement coupé, notamment beaucoup de références savantes qui peut-être 'ne parlaient plus' aux lecteurs de la fin du XVIII ${ }^{e}$ siècle 
avaient été supprimées. De même le langage avait été actualisé par la traduction, là où par exemple country devenait patrie, ou men citoyens, etc. Toutes ces éditions successives attestent l'intérêt pour cet ouvrage, un intérêt que l'on peut retrouver dans certaines interventions des conventionnels lors du procès de Louis XVI.

Comme le député Rülh l'avait remarqué, la Défense de Milton présentait des justifications théoriques à l'appui de la légitimité du jugement du souverain. En effet, tout au long du mois de novembre jusqu'au début de décembre, la question débattue concernait encore l'inviolabilité et donc la légitimité de juger le «ci-devant » roi. C'est dans ce sens-là que l'ouvrage de Milton fut rappelé par deux députés, résolument favorables au jugement de Louis XVI, afin de faire avancer et accélérer un procès qui procédait très lentement. Il s'agit de l'opinion de François-Siméon Bézard, député du département de l'Aisne, qui affirmait: "Je ne vous entretiendrai pas de l'inviolabilité ; tout ce que je pourrais dire contre cette divinité de la tyrannie ne serait qu'une répétition des principes de la doctrine de Milton, dans sa défense du peuple anglais contre Saumaize; et ils ont été suffisamment développés à la tribune ${ }^{26} »$; et de celle Marc-Guillaume Vadier, du département de l'Ariège, qui s'exprimait d'un même ton : "Cette inviolabilité burlesque est donc une monstruosité dans l'ordre moral, comme dans l'ordre politique ; elle ne peut servir de bouclier au crime, et à la plus lâche des trahisons ; c'est le comble de l'absurdité, que de vouloir l'étendre audelà des délits d'administration, et des fautes commises dans l'exercice de la royauté. Il serait inutile de répéter tout ce qui a été dit par Milton, pour réfuter le dogme de cette inviolabilité liberticide... Mais peut-on ne pas s'élever contre ces lâches profanateurs des Droits de l'homme qui ont eu recours à ce talisman pour déifier leur idole ${ }^{27}[. .]$.$» Du point de vue des députés qui soutenaient la cause du procès, les$ argumentations de Milton étaient opérationnelles pour surmonter l'impasse posée, dans la discussion de novembre 1792, par la question de l'inviolabilité établie par la Constitution de 1791.

C'était aussi l'avis, en dehors de la Convention, du Conseil du Département de la Drôme qui, au cours de la séance publique du 14 novembre 1792, accueillit la pétition pour faire réimprimer l'écrit de John Milton intitulé Défense du peuple anglais, sur le jugement et la condamnation de Charles premier, roi d'Angleterre, nouveau titre donc du même ouvrage, suivi par un sous-titre très éloquent: "Ouvrage propre à éclairer sur la circonstance actuelle où se trouve la France ${ }^{28}$.» Le Conseil après lecture de quelques extraits, considérait que «l'ouvrage de Milton établit et développe avec autant de clarté que de solidité, les droits imprescriptibles de la souveraineté de tous les peuples... que le génie qui l'a produit embrase tous les cœurs du feu sacré de la liberté, qu'il présente des idées justes et saines de la royauté...; qu'il démontre aux partisans de l'inviolabilité des rois, que dans tous les tems et chez toutes les nations leurs crimes ont été expiés par l'échafaud; qu'il est du devoir des Administrateurs de former et de mûrir l'opinion publique, sur la grande question qui s'agite à la Convention nationale pour le jugement de Louis Capet lequel doit être présenté à la sanction du Peuple; que l'Administration est sûre de remplir cet objet essentiel en répandant, sur-tout dans le campagnes, la connoissance d'un livre devenu très-rare, et par la lecture duquel tout Français Républicain pourra déméler avec sagacité les rapports et l'analogie qui existent entre la conduite de Charles Stuard et celle de Louis Capet $^{29}$. " Le Procureur-Général-Syndic arrêtait donc l'impression de l'ouvrage de Milton « au nombre de mille exemplaires aux frais des Administrateurs présens à la Séance», et l'envoi d'exemplaires à toutes les 
Communes, aux Départements, et la Convention Nationale, qui étaient invités à ordonner la réimpression dans toute l'étendue de la République.

Par cet arrêté, le Conseil départemental de la Drôme ${ }^{30}$ entendait participer au débat public concernant le sort de Louis XVI, et promouvait par conséquent la lecture de Milton afin de persuader que le procès (et la condamnation à mort) était un acte de justice nationale. La nouvelle édition de la Défense de Milton, fut présentée à l'attention de la Convention nationale au cours de la séance du 27 décembre 1792, par l'un des député de la Drôme, Marc-Antoine Jullien: "J'ai l'honneur de déposer comme hommage, sur le bureau de la Convention, au nom des administrateurs du département de la Drôme, un exemplaire d'un ouvrage intitulé : Défense du peuple anglais sur le jugement de Charles I ${ }^{e r}$, roi d'Angleterre par Milton, dont les administrateurs de la Drôme ont ordonné la réimpression à leurs frais ${ }^{31}$. " La Convention nationale accepta l'hommage de cet ouvrage et ordonna qu'une mention honorable fût faite à l'adresse des administrateurs de la Drôme dans le procès-verbal.

Dès que la Convention décida de juger Louis Capet, et de le faire paraître à sa barre le 11 décembre, les interventions déjà nombreuses des particuliers faisant imprimer leur texte pour participer au débat dans l'espace public, et surtout pour défendre la cause de Louis XVI, se multiplièrent. Au moment où il s'agissait de défendre le roi, la référence à l'histoire d'Angleterre n'avait pas perdu d'efficacité argumentative, comme déjà l'avait montré, le premier, Jacques Necker dans ses Réflexions. Pourtant, le pamphlet anonyme Au Peuple souverain, Sur le procès de Louis Seize, signé par un Républicain ${ }^{32}$, utilisait l'exemple du procès de Charles $\mathrm{I}^{\mathrm{er}}$ pour plaider à la fois l'innocence de Louis et le salut de la République. En mettant en garde «le bon François » sur les « usurpateurs » se cachant derrière ceux qui voulaient voir couler le sang de Louis XVI, l'auteur rappelait les dérives despotiques du cas anglais : «Cromwel, et je ne cesserai de te le rappeler, Cromwel, te disje, par ses sombres complots, fit décapiter Charles. Ses fautes, que Louis n'a jamais commises, sembloient excuser son supplice. Le lendemain de son exécution, chacun fouillant en sa conscience, se trouvoit coupable de l'avoir toléré. Quel en a été le fruit ? le renversement d'une république qu'on croyoit éternelle; le massacre des juges et de leurs complices ; des séditions, la perte de la liberté, car celle dont jouit l'Angleterre, un souffle peut la renverser ${ }^{33}$. " Le recours à l'appel au peuple par l'institution d'une haute cour de justice nationale composée par deux hommes «d'une vie sans tache et sans reproche, patriotes reconnus depuis la révolution, choisis par chaque département, c'était la seule solution envisageable pour prévenir le despotisme dont l'histoire anglaise avait donné une leçon éloquente.

Après le plaidoyer de la défense, le 26 décembre, le procès commença une nouvelle phase, celle de la discussion qui devait amener à la sentence. Jusqu'à ce moment, comme on l'a $\mathrm{vu}$, la référence à l'histoire d'Angleterre avait accompagné le débat public, dans les opinions des députés, dans les interventions de particuliers au moyen de l'impression, aussi bien que dans la presse. L'exemple était relaté, utilisé pour ou contre le jugement, mais, tout en demeurant opérationnel, il n'était pas véritablement passé au crible de la critique afin d'en évaluer sa validité. C'est dans un article paru dans les Annales patriotiques et littéraires du 31 décembre qu'un journaliste bien avisé sur l'histoire d'Angleterre, Jean-Baptiste Salaville, faisait le point sur cette comparaison constante des deux événements historiques, le jugement de Charles Stuart et celui de Louis Capet :

On cherche continuellement des rapports entre notre position actuelle et celle où se trouvoit l'Angleterre à la mort de Charles Stuart. On compte pour rien la différence des 
temps et des circonstances : on ne voit pas que notre situation intérieure et extérieure n'a rien en commun avec celle de l'Angleterre à cette époque ; les despotes de l'Europe ne se crurent point intéressés à se coaliser pour empêcher la mort de Charles, parce que la royauté n'étoit point universellement attaquée. Les principes de la souveraineté du peuple, professé par Milton et par quelques autres républicains, n'avoient pas passé sur le continent; renfermés dans la Grande-Bretagne, ils n'y étoient pas même devenus populaires : aujourd'hui nous devons nous attendre à voir tous le rois défendre Louis par tous les moyens possibles, parce qu'il s'agit de la royauté. [...]

Si nous apportons des délais interminables à ce jugement, nos ennemis réuniront tous leurs effors et nous feront une guerre sanglante pour conjurer ce jugement; quand il sera prononcé, ils n'auront plus ce motif, ils ne se mettront plus en frais pour l'empêcher. On nous parle encore des dangers intérieurs, et on n'oublie pas de s'autoriser du rappel des fils de Charles. Sans doute si nous devons avoir un Cromwell, si après avoir décrété la république nous ne l'organisons pas, si nous ajournons éternellement la constitution, si de misérables querelles consument toutes les délibérations de nos représentans, nous reviendrons infailliblement à la monarchie ; mais ce ne sera par la mort de Louis qui nous y aura ramené, ce seront d'autres causes, et nous aurons bien mérité de perdre tout le fruit de nos sacrifices, puisque nous n'auront pas voulu être libres ${ }^{34}$.

21 Salaville, comme on l'a vu, avait été le traducteur de l'ouvrage de Milton parut au commencement de la Révolution. Son analyse de cette comparaison naissait d'une connaissance profonde du texte de l'auteur de la Defensio pro populo anglicano et de l'histoire anglaise. Il fallait bien considérer les deux contextes ( « on compte pour rien la différence des temps et des circonstances») pour en tirer les justes conclusions. Par conséquent, juger Louis XVI d'après l'exemple anglais pouvait se révéler une démarche trompeuse si l'on se fixait, comme tout le monde semblait le faire, sur «la situation intérieure et extérieure » sans considérer aucunement les différences de contexte. C'était, au fait, une comparaison sans perspective historique, quoique la crainte d'un Cromwell français et le retour de la monarchie se profilent en contrepartie aussi pour le procès de Louis XVI.

En effet, cette admonition provenant d'une lecture de l'histoire, notamment les conséquences potentiellement néfastes qui suivraient à la condamnation à mort, jouèrent un rôle déterminant chez certains membres de la Convention au moment de répondre à la troisième question des appels nominaux, à savoir «quelle peine Louis, ci-devant roi des Français, a-t-il encourue?", dans les séances du 16-17 janvier 1793. Parmi ceux qui ne votèrent pas pour la mort de Louis, l'exemple de l'histoire d'Angleterre était la justification, fondée sur l'expérience, voir enseignement, du passé sur laquelle se fondait le vote. C'est le cas du député du Loiret Michel Gentil qui, en votant pour la réclusion jusqu'à la paix et ensuite la déportation, affirmait que: «L'histoire d'Angleterre en me présentant un cas très pareil à celui-ci, m'a porté à faire de terribles réflexions. Je ne veux donc pas que mon opinion contribue à donner à la France un Cromwel, ou le retour imprévu de Charles $\mathrm{II}^{35}$.»; ou de Mollevant, député du Meurthe, qui opta pour la détention, convaincu que «la mort de Louis le dernier ne serait pas moins inutile à la liberté des Français que le furent la mort de César à celle des Romains, la mort de Charles Ier à la liberté anglaise ${ }^{36}$ "; ou encore de Guyomar, député du département de la Côte du Nord, qui s'exprima pour la détention pendant la guerre et le bannissement à la paix : «Si la mort d'un individu jadis roi tuait la royauté; si l'Angleterre, la Turquie ne nous fournissaient pas des exemples contraires, le problème serait bientôt résolu ; mais 
un roi décapité, remplacé par un protecteur, auquel succéda un roi, fils de Charles décapité. Cet exemple d'une République éphémère dans l'île britannique vaut bien la peine d'être considérée ${ }^{37}$.» L'histoire anglaise, donc, pour certains parmi ceux qui n'avaient pas voté la mort de Louis XVI, était un exemple à ne pas suivre si l'on souhaitait que la République vive et dure.

Louis XVI fut pourtant condamné à mort et le matin du 21 janvier 1793 la lame de la guillotine tomba sur sa tête devant une foule silencieuse réunie place de la Révolution. A l'occasion de l'exécution de Louis XVI, le journal hebdomadaire Les Révolutions de Paris, consacra une grande partie du commentaire de la journée à la comparaison entre la mort de Charles Stuart et celle de Louis Capet. Le journal de Prudhomme insistait sur ce parallèle afin de montrer comment toutes les limites constatées dans l'exemple historique anglais avaient été surmontées par le jugement de la Convention nationale : «L'exécution de Louis XVI a presque été l'anniversaire de celle de Charles premier, roi d'Angleterre [...] L'affaire de Charles premier, quoique poursuivie d'une manière bien moins légale que celle de Louis, avoit cependant un autre caractère de grandeur. (...) En Angleterre, la royauté fut conduite à l'échafaud avec tout son cortège. [...] Pour Louis XVI, il est mort comme le plus vil des criminels ; ce n'est pas la royauté que l'on a puni en lui, mais le coupable ${ }^{38}$."

Procès à un homme, procès à la royauté, cette discordance de vision dans les pages des journaux, on l'a vu, s'était articulée également sur la comparaison avec le procès de Charles Stuart. S'il s'agissait de la royauté, comme l'avait estimé Salaville dans les Annales patriotiques et littéraires, il fallait craindre une coalition internationale des têtes couronnées contre la France. Si, comme l'interprétait le rédacteur des Révolutions de Paris, on avait condamné et puni un homme coupable, un tyran, alors il fallait invoquer l'union générale de la nation comme le seul salut de la République: «A présent ne craignons donc rien que nous-mêmes; notre salut est dans nos mains. Le tyran n'est plus [...]. Répétons tous les serments que la convention entière a prêté sur le corps sanglant de Pelletier, cette dernière victime de la tyrannie : jurons d'être toujours unis, jurons le salut de la patrie; car l'union fait notre force, et c'est de notre union que dépendra la conservation de la république ${ }^{39}$.»

25 En effet, un jour avant l'exécution de Louis XVI, le 20 janvier, Le Pelletier de SaintFargeau qui avait voté pour la mort du roi, avait été assassiné par un royaliste voulant venger la condamnation de Louis XVI. Dans les discours prononcés à la Convention sur l'assassinat de l'un de ses membres, Saint-Fargeau fut tout de suite assimilé aux martyrs de la liberté qui l'avaient précédé et intégré dans cet imaginaire héroïque ${ }^{40}$. Ainsi, le 21 janvier, Barère demanda aux membres de la Convention de rendre hommage «à un véritable martyr de l'opinion républicaine : car l'homme qui a le courage de voter pour la mort des tyrans est un véritable républicain ${ }^{41}$. » Au cours de la séance du jour suivant, Marie-Joseph Chénier inscrivait le nom de Le Pelletier dans la liste glorieuse de ceux qui avaient péri pour la liberté : «Citoyens, vous léguerez à la postérité de grands souvenirs et de grands exemples; [...] Lepelletier, immortalisé par son assassin, vous montre la palme civique des martyrs de la liberté. Il vient de prendre place entre Barneveldt et Sidney. Son sort paraîtra digne d'envie à tous les vrais républicains; et les honneurs dont vous récompensez sa mémoire lui donneront des successeurs qui, comme lui, comme vous, citoyens, sauront tout sacrifier à la nation souveraine, et dans les circonstances les plus graves, environnés de périls et d'orages, considéreront toujours la liberté le devoir, et jamais la vie ${ }^{42}$.» Le Pelletier Saint-Fargeau, devenait comme Algernon Sidney, l'un des 
grands martyrs de la liberté, son sacrifice étant assimilé à celui du penseur républicain anglais mort sur l'échafaud en 1683.

Les Discourses Concerning Government de Algernon Sidney, publiés de façon posthume en 1698, et traduits en français en 1702, exercèrent une influence durable tout au long du XVIII ${ }^{\mathrm{e}}$ siècle, en Europe aussi bien que dans le continent américain ${ }^{43}$. Ils furent également édités à Paris, en l'an II. Parallèlement à son œuvre, la biographie emblématique de Sidney incarnait un système de valeurs, une adhésion totale à la cause républicaine. La seule invocation de son nom avait le pouvoir d'animer et de nourrir les esprits républicains, de rappeler ce que servir la liberté pouvait signifier, en termes d'engagement et de prix à payer, c'est-à-dire le sacrifice le plus haut, celui de la vie, comme l'avait évoqué Robespierre lors de son discours du 28 décembre 1792, contre la proposition de soumettre aux assemblées primaires le jugement de Louis XVI ${ }^{44}$.

En effet, les penseurs républicains anglais du XVII ${ }^{e}$ siècle furent, dans la plupart de cas, impliqués dans les luttes partisanes, leurs valeurs provenant non seulement des idées qu'ils avaient théorisées mais de l'engagement actif dans la vie politique du pays. Milton incarne sans aucun doute la référence la plus importante du républicanisme anglais dans la période du procès de Louis XVI. La correspondance des événements historiques, le thème du régicide, la Défense du peuple anglais contribuait à alimenter le débat et la référence résidait aussi dans le prestige et dans la foi républicaine attribuée à l'auteur. Ainsi, lors de l'assassinat de Le Pelletier, la figure de Sidney était régulièrement convoquée dans les discours pour donner corps à la nouvelle mythologie républicaine, celle d'une fraternité sans limites dans l'espace et dans le temps ${ }^{45}$.

L'histoire d'Angleterre du XVII ${ }^{\mathrm{e}}$ siècle, le procès de Charles I $^{\mathrm{er}}$, la dictature cromwellienne et la restauration de la monarchie qui suivit, hanta les opinions, les discours tout au long du jugement de Louis XVI. Au cours de ce procès, le passé britannique représenta un modèle à adopter, à améliorer, voir surmonter, ou à réfuter; il servit de clé pour interpréter le présent, d'admonition pour déterminer l'action et par là le futur. Il fut indistinctement évoqué par ceux qui voulaient sauver le roi, aussi bien que par ceux qui voulaient dès le début le condamner. Pour ces derniers, la récupération des penseurs du républicanisme classique comme Milton et Sidney, contribua à alimenter ce lien avec le passé, à donner des justifications théoriques à l'appui du jugement et servit enfin à créer une continuité dans la rédaction d'un nouveau chapitre de l'histoire républicaine.

\section{NOTES}

1. Michael WALZER, Régicide et Révolution. Le procès de Louis XVI, Traduit de l'américain par J. Debouzy, Suivi de Discours au procès de Louis XVI, réunis et annotés par A. Kupiec, et Régicide et Terreur, controverse F. Fehér - M. Walzer, Traduit de l'américain par A. Kupiec, Paris, Payot, 1989 (éd. originale 1974); Olivier LUTAUD, Des Révolutions d'Angleterre à la Révolution française. Le tyrannicide \& Killing no Murder (Cromwell, Athalie, Bonaparte). Essai de littérature politique comparée, La Haye, Martinus Nijhoff, 1973.

2. La feuille villageoise, $\mathrm{n}^{\circ}$ 51, jeudi 27 septembre 1792, p. 599 . 
3. C'était l'une des maximes du juriste anglais Bracton (De legibus et consuetudinis angliae) reprise par ceux qui affirmaient la théorie de la souveraineté populaire depuis les Vindiciae contra Tyrannos et cité par John Milton dans sa Defensio pro populo anglicano ou Sidney dans ses Discourses on Government.

4. Révolutions de Paris dédiées à la Nation, n 169, Du 29 septembre au 6 octobre 1792, p. 55-56.

5. Voir la nouvelle de sa parution dans rubrique des Livres nouveaux de la Gazette Nationale ou le Moniteur universel, $\mathrm{n}^{\circ}$ 302, dimanche 28 octobre 1792, p. 312.

6. Histoire entière et veritable du procez de Charles Stuart, Roy d'Angleterre. Contenant, en forme de Journal, tout ce qui s'est faict et passé sur ce sujet dans le Parlement, et en la Haute Cour de Justice; Et la façon en laquelle il a esté mis à mort. Au mois de Janvier, 1648. A quoy sont adjoustées Quelques Declarations du Parlement publiées auparavant, pour faire voir plus amplement, quels peuvent avoir esté les motifs et raisons d'une procedure si extraordinaire. Le tout fidelement receuilly des pieces Authentiques et traduit de l'Anglois, A Londres, Imprimé par J. G., 1650. Cet ouvrage était précédé par un avis au lecteur dans lequel le traducteur avouait avoir voulu, par son recueil, satisfaire la vérité aussi bien que la curiosité du monde.

7. Histoire entière et véritable du procez de Charles Stuard, Roi d'Angleterre. Contenant, en forme de Journal, tout ce qui s'est faict et passé sur ce sujet dans le Parlement, et en la Haute Cour de Justice; Et la façon en laquelle il a été mis à mort. Au mois de Janvier, 1648 et 49 . Le tout fidellement receuilly des pièces autentiques et traduit de l'Anglois, A Paris, Réimprimé par Chaudrillié, rue de Rohan $n^{\circ} 16$, sur l'édition de J. G. imprimée à Londres en 1650, Préface de l'Éditeur. Cet ouvrage eut une seconde édition qui, à la différence de la première ne comportait aucune préface. Dans les Annales patriotiques et littéraires, $\mathrm{n}^{\circ} 345$, du 10 décembre 1792, p. 1542 on lit, l'annonce de la parution de cette nouvelle édition : «La première édition ayant été épuisée en fort peu de temps, l'éditeur en offre une nouvelle réimpression. »

8. Relation véritable de la mort cruelle et barbare de Charles I, Arrivée à Londres le huitième Février mil six cent quarante-neuf. Avec la Harangue fait par Sa Majesté sur l'échafaud. Traduite de l'Anglais en Français par J. Ango, sur l'imprimé à Londres chez F. Coles, Réimprimée A Paris, Par Lepetit, 1792. Ce recueil contenait les pièces suivantes originairement publiées entre 1649 et 1650 : Relation véritable de la mort barbare et cruelle de Charles I ${ }^{\text {er }}$, roi d'Angleterre. Arrivée à Londres le huictiesme Fevrier mil six cens quarente-neuf, A Paris, Chez Robert Feugé, 1649 ; Arrêt de mort donné contre Sa Majesté britannique par les parlementaires d'Angleterre..., publié en 1649 sous le titre : Récit veritable de tout ce qui s'est fait au procez du roy de la Grand'Bretagne, son arrest et la manière de son exécution; avec la harangue faite par Sadite Majesté sur l'eschaffaut. Traduit d'anglois en françois par I. Ango, interprète de ladite langue, sur l'imprimé à Londres par François Coles, 1649 ; Sommaire de ce qui s'est passé de plus memorable en Angleterre, Depuis l'année 1640 iusques au premier Ianuier 1650. Contenant la Convocation du Parlement, les causes et les effets des troubles, les differences de factions, le procez fait au roy, sa condemnation et son execution de mort, ce qui a esté fait pour l'establissement d'une Republique, et les Partys qui sont maintenant dans cet Estat, A Paris, chez Pierre Lepetit, Imprimeur et Libraire ordinaire du Roy, 1650.

9. L'Angleterre instruisant la France, Tableau historique et politique du règne de Charles I ${ }^{\text {er }}$ et de Charles II. Servant d'introduction à la Relation de la mort cr... et b... de Charles Ier, suivie de sa harangue sur l'échaffaud, A Londres, Et se trouve à Paris, Chez Lepetit, 1793. Voir Olivier LUTAUD, Des Révolutions d'Angleterre à la Révolution française, op. cit., p. 227 ; Olivier LUTAUD, «Emprunts de la Révolution française à la première révolution anglaise. De Stuart à Capet, de Cromwell à Bonaparte ", Revue d'histoire moderne et contemporaine, XXXVII, octobre-décembre 1990, p. 601-602.

10. L'Angleterre instruisant la France, op. cit., p. 96.

11. Archives parlementaires, op. cit., t. LIII, p. 8.

12. Ibidem, p. 9. 
13. Archives parlementaires de 1789 à 1860 . Recueil complet des débats législatifs et politiques des chambres françaises, t. LIII, 27 octobre 1792 au 30 novembre 1792, Paris, Paul Dupont Éditeur, 1898, p. 280.

14. "Jamais ce célèbre publiciste, qui serait le premier des hommes s'il n'avait pas prostitué sa plume à l'apologie de la monarchie et de la noblesse, n'aurait eu le prétexte de dire "ce fut un assez beau spectacle de voir les efforts impuissants des Anglais pour rétablir parmi eux la République, de voir le peuple étonné cherchant la démocratie et ne la trouvant nulle part, de le voir enfin, après bien des mouvements, des chocs et des secousses, forcé de se reposer dans le gouvernement même qu'il avait proscrit" ». Archives parlementaires, t. LIII, op.cit., p. 280. Sur la représentation du modèle anglais dans l'Esprit des lois de Montesquieu, voir Édouard TILLET, La constitution anglaise, un modèle politique et institutionnel dans la France des Lumières, Aix-en-Provence, Presses Universitaires d'Aix-Marseille, 2001, p. 249-291

15. Laurence L. BONGIE, David Hume, Prophet of the Counter-revolution, Oxford, Clarendon Press, 1965. Voir notamment le chapitre III From 1789 to the Trial of Louis XVI, et le chapitre IV The Trial of "le Stuart Français". Michel MALHERBE, Hume's Reception in France, dans Peter Jones (dir.), The Reception of David Hume in Europe, London-New York, Thoemmes Continuum, 2005, p. 43-97. Sur l'Histoire d'Angleterre p. 77-86.

16. Laurence L. BONGIE, David Hume, op. cit., p.121. Vers la fin de novembre 1792, Louis XVI, adressait au Conseil général de la Commune une liste d'ouvrages qu'il désirait se procurer : des auteurs latins classiques (César, Salluste, Suétone, Cornelius-Nepos, Tite Live), mais aussi les Métamorphoses d'Ovide ou les fables de Phèdre et de La Fontaine, destinés à l'éducation de son fils. Même si le Conseil général lui accorda la demande, certains membres disaient qu'il n'aurait pas eu assez de temps pour tout lire ; d'autres pensaient qu'il n'aurait pas compris certains ouvrages, "plusieurs vouloient qu'on lu !i donnât en place les révolutions d'Angleterre, celles d'Amérique, la vie de Cromwell» (Suite du Journal de Perlet, $n^{\circ} 66$, lundi 26 novembre 1792, p. 525. Voir aussi le n 332 du mardi 27 novembre 1792 du Moniteur, dans la rubrique consacrée aux nouvelles de la Commune, séance du 23 novembre, p. 570).

17. Jacques NECKER, Réflexions présentées à la nation française, sur le procès intenté à Louis XVI. Par M. Necker, A paris, chez Volland, 1792, p. 30

18. Jacques-Pierre BRISSOT, Discours sur la question de savoir si le roi peut être jugé, Prononcé à l'Assemblée des Amis de la Constitution, dans la Séance du 10 Juillet 1791, p. 10 e note 2 : « ... jamais les Anglois n'ont cru que leur Prince fût inviolable lorsqu'il vouloit bouleverser la Constitution par des manœuvres ou par la force. Lisez Locke, Sidney, Milton, Macaulay... » Voir Laurence L. BONGIE, David Hume, op. cit., p. 113-119.

19. Le traducteur de l'Histoire de Macaulay était Charles-Philippe-Toussaint Guiraudet, à son tour auteur d'un Précis de toute l'Histoire d'Angleterre jusqu'à l'avénement de Jacques Ier.

20. Catherine Macaulay GRAHAM, Histoire d'Angleterre; depuis l'avènement de Jacques I, jusqu'à la révolution. Traduite en Français, et augmentée d'un discours préliminaire, contenant un précis de toute l'histoire d'Angleterre, jusqu'à l'avènement de Jacques I : et enrichie de notes par Mirabeau, A Paris, Chez Gattey, 1791. Dans le Moniteur du 9 octobre 1791, n²82, p. 62, on lit : « cet ouvrage, l'un des plus importants que l'on ait entrepris depuis la révolution, et qui obtiendra sûrement en France le brillant succès dont il jouit en Angleterre ». Voir Olivier LUTAUD, Des Révolutions d'Angleterre à la Révolution française, op. cit., p. 226. Bridget HILL, The Republican Virago. The Life and Times of Catharine Macaulay, Historian, Oxford, Clarendon Press, 1992. Voir également Marie-Odile BERNEZ, «Catharine Macaulay et Mary Wollstonecraft. Deux femmes dans le débat sur la Révolution française en Angleterre ", Annales historiques de la Révolution française, 344, 2, 2006, p. 161-178.

21. Gazette Nationale ou le Moniteur universel, $\mathrm{n}^{\circ} 45$ p. 375-376. Les tomes 3, 4, 5 parurent au mois d'octobre 1792. Voir l'annonce dans le Moniteur, $n^{\circ} 302$, Du dimanche 28 octobre 1792, p. 312. Le procès de Charles Stuart se trouve notamment dans le cinquième tome, p. 217-238. 
22. Catherine Macaulay GRAHAM, Histoire d'Angleterre, op. cit., t. I, p. ix.

23. Joannis mILtoni, Defensio pro populo anglicano: Contra Claudii Anonimi, alias Salmasii, defensionem regiam, Londini, Typis Du Gardianis, 1651; John MiLToN, A Defence of the People of England. In Answer to Salmasius's Defence of the King, s.l., 1695.

24. Ibidem, p. 3 « put to death a good, nor a just, nor a merciful, nor a devout, nor a godly, nor a peaceble King, as you stile him; but an Enemy, that has been so to us almost ten years to an end; nor one that was a Father, but a Destroyer of his Country. "

25. Raymonde MONNIER, "Traduction, transmission et révolution: enjeux rhétoriques de la traduction des textes de la conception républicaine de la liberté autour de 1789 ", Annales historiques de la Révolution française, 364, avril-juin 2011, p. 40-47 ; Olivier LUTAUD, Des Révolutions d'Angleterre à la Révolution française. L'exemple de la liberté de la presse ou comment Milton "ouvrit » les États-Généraux, dans Christian CROISILLE, Jean EHRARD (dir.), La légende de la Révolution. Actes du colloque international de Clermont-Ferrand (juin 1986), Clermont-Ferrand, Centre de recherches révolutionnaires et romantiques, 1988, p. 115-125; Christophe TOURNU, Milton, Mirabeau : rencontre révolutionnaire, Paris, EDIMAF, 2002 (cet essai contient également l'édition des ouvrages de Milton : Sur la liberté de la presse, imité de l'anglois de Milton par Mirabeau l'aîné et Défense du peuple, sur le jugement et la condamnation de Charles Premier, Roi d'Angleterre); Raymonde MONNIER, Républicanisme, patriotisme et Révolution française, Paris, L'Harmattan, 2005, p. 204-205 ; François QUASTANA, La pensée politique de Mirabeau (1771-1789) : "républicanisme classique" et régénération de la monarchie, Aix-en-Provence, Presses universitaires d'Aix-Marseille, 2007, p. 557-559.

26. Archives parlementaires, op. cit., t. LIV, $1^{\text {er }}$ décembre 1792 au 10 décembre 1792, p. 112.

27. Ibidem, p. 334.

28. Défense du peuple anglais, sur le jugement et la condamnation de Charles premier, roi d'Angleterre, par Milton. Ouvrage propre à éclairer sur la circonstance actuelle où se trouve la France. Réimprimé aux frais des Administrateurs du Département de la Drôme, a Valence, chez P. Aurel, Imprimeur du Département de la Drôme, 1792.

29. Ibidem, fo $3 \mathrm{r}-3 \mathrm{v}$. La phrase «lequel doit être présenté à la sanction du Peuple » a été rayée et rendue presque illisible dans l'exemplaire conservé à la Bibliothèque Nationale de France.

30. Michel SEYVE, Les montagnards drômois dans la crise de l'été 1793 : "la Drôme a bien mérité de la patrie ", dans Michèle NATHAN-TILLOY, Claude GENTY (dir.), Les drômois acteurs de la Révolution, Actes du colloque de Valence Hôtel du Département 12, 13, 14 octobre, Valence, Archives départementales de la Drôme, 1990, p. 416-418. Il faut noter que parmi les membres du Conseil, siégeaient les frères Joseph et surtout Claude (François de) Payan qui ensuite, par l'intermédiaire de Jullien, député à la Convention, connut Robespierre et en devint l'un des plus fervents admirateur. Après une carrière rapide en tant que agent national de la Commune de Paris, il périra sur l'échafaud le 9 Thermidor.

31. Archives parlementaires, op. cit., t. LV, 11 décembre 1792 au 27 décembre 1792, , p. 705.

32. Ce pamphlet est attribué par le catalogue de la Bibliothèque Nationale de France à Brissot.

33. A Paris, Chez Lepetit, Libraire, quai des Augustins, $n^{\circ} 32$, p. 25.

34. Annales patriotiques et littéraires, $n^{\circ}$ CCCLXVI, Du lundi 31 Décembre 1792, p. 1638-1639. Sur Salaville journaliste des Annales patriotiques voir Michael L. KENNEDY, «L'oracle des jacobins des départements » : Jean-Louis Carra et ses « Annales patriotiques », dans Albert soBoul (dir.), Actes du Colloque Girondins et Montagnards (Sorbonne, 14 décembre 1975), Paris, Société des Études Robespierristes, 1980, p. 250.

35. Archives parlementaires, op. cit., t. LVII, 12 janvier 1793 au 28 janvier 1793, p. 353.

36. Ibidem, p. 358. Il faut citer également Doublet du département de la Seine-Inférieure; «Intimement convaincu des attentas de Louis, mais pénétré encore du malheurs dont a été pour l'Angleterre la mort de Stuart, je vote pour la détention et le bannissement après l'affermissement de la République. » (p. 375) ; et Bancal, « Parce que l'histoire d'Angleterre donne 
une grande leçon à tous les peuples qui fondent des Républiques » détention et bannissement (p. 367).

37. Ibidem, p. 400.

38. Révolutions de Paris, dédiées à la nation, $\mathrm{n}^{\circ} 185$, du 19 au 26 janvier 1793, p. 211-212, 213. Sur Les révolutions de Paris, Jean TULARD, Jean-François FAYARD, Alfred FIERRO, Histoire et dictionnaire de la Révolution française 1789-1799, Paris, Laffont, 1987, p. 1051, 1067 ; Claude BELLANGER, Jacques GODECHOT, Pierre GUIRAL, Fernand TERROU, Histoire générale de la presse française, t. 1, Des origines à 1814, Paris, Presses Universitaires de France, 1969, p. 451-453.

39. Révolutions de Paris, dédiées à la nation, $\mathrm{n}^{\circ} 185$, du 19 au 26 janvier 1793, p. 220.

40. Voir Antoine SCHNAPPER, A propos de David et des martyrs de la Révolution, dans Michel VovelLE ( dir.), Les images de la Révolution. Actes du colloque des 25-26-27 octobre 1985 tenu en Sorbonne, Paris, Publications de la Sorbonne,1988, p. 109. Voir également sur le «martyr » Le Pelletier, Antoine DE BAECQUE, « Le corps meurtri de la Révolution. Le discours politique et les blessures des martyrs (1792-1794) », Annales historiques de la Révolution française, 267, 1987, p.17-41; Rolf REICHARDT, Hubertus коHLE, Visualizing the Revolution. Politics and the Pictorial Arts in Late Eighteenth-Century France, London, Reaktion Books, 2008, p. 161-164.

41. Ibidem, p. 522.

42. Archives parlementaires, op. cit., t. LVI, p. 542.

43. Jonathan sсотT, Algernon Sidney and the English Republic 1623-1677, Cambridge, Cambridge University Press, 1988; ID., Algernon Sidney and the Restoration Crisis, 1677-1683, Cambridge, Cambridge University Press, 1991; Alan Craig Houston, Algernon Sidney and the Republican Heritage in England and America, Princeton, Princeton University Press, 1991.

44. Archives parlementaires, op. cit., t. LVI, p. 22.

45. Voir pour la question Républicanisme et régicide : Blair WORDEN, « Republicanism, Regicide and Republic: The English Experience », dans Martin VAN GELDEREN, Quentin Skinner (dir.), Républicanism: a shared European heritage, vol. 1, Cambridge, Cambridge University Press, 2002, p. 307-328.

\section{RÉSUMÉS}

Dès le début de l'instruction du procès de Louis XVI jusqu'à son exécution, la référence à l'histoire anglaise, notamment au jugement de Charles $I^{\text {er }}$ et à l'ascension au Protectorat de Cromwell, représenta un passage fréquent dans les discours des contemporains. Les opinions des conventionnels aussi bien que celles des particuliers et des journalistes renvoyaient souvent à l'exemple historique du procès de Charles Stuard, à l'appui ou à l'encontre de la légitimité du jugement. Dans ces discours on retrouvait parfois la référence aux figures les plus emblématiques du républicanisme anglais du XVII ${ }^{\mathrm{e}}$ siècle, notamment celles de John Milton et Algernon Sidney. Le premier était cité en tant qu'auteur d'une Défense du peuple anglais contre les accusations de régicide, ouvrage réédité $a d$ hoc en français à l'époque du procès de Louis XVI ; le second en tant que martyr de la liberté, son seul nom étant capable d'évoquer l'engagement et le sacrifice extrêmes pour la cause républicaine.

Since the beginning of the trial of Louis XVI until his execution, the reference to the English history (from the proclamation of the Commonwealth to the Cromwell's ascent to power) 
represented a recurring passage in contemporary speeches. The opinions of representatives to national Convention as well as those of private citizens or journalists often referred to the example of the trial of Charles I, pro or against the legitimacy of the judgement. In these speeches the mention to English republicanism emblematic figures, such as John Milton and Algernon Sidney, was also given. Milton was quoted because of his Defence of the people of England, an answer he wrote in response to the accusation of regicide, and reprinted in French during the trial of Louis XVI. Sidney was evoked because of his symbolic meaning as a martyr of Liberty.

INDEX

Mots-clés : Convention nationale, procès, roi, Louis XVI, Charles Ier, John Milton, Angleterre, républicanisme

Keywords : National Convention, Trial, King, Louis XVI, Charles I, John Milton, England, Republicanism

\section{AUTEUR}

\section{CLIZIA MAGONI}

Université de Caen 